\title{
Reserve Management in Restructured Power System Considering Loads Reliability
}

\author{
Saeed Zolfaghari, Mohammad H. Hashemi, and Azam Zolfaghari
}

\begin{abstract}
Determining the spinning reserve capacity is amongst the most important tasks of system operator to insure safe and reliable operation of power system. So far, various methods are proposed to determine reserve capacity which are based on deterministic or probabilistic criteria. Due to considering the stochastic nature of system events, probabilistic approaches are more preferable in comparison to deterministic approaches. In practice, the value of interrupted loads is not the same for all consumers and some of them like industrial ones have higher value of lost load (VOLL). Thus, consumes with higher values of lost load are willing to pay more in order to gain higher reliability levels than consumers with less VOLL. In other words, they are more concerned with their individual load point reliability. In this paper a new method is presented to optimally determine the system spinning reserve requirement in a competitive simultaneous energy and reserve market render based on cost-benefit analysis and stochastic programming technique is proposed considering different values of lost load at each bus. Including units and lines outages and their corresponding likelihoods in the objective function, regard for loading rate of the generators constraints and transmission flow constraints are the advantages of the proposed method. Simulations performed on the 14-bus IEEE test system demonstrate effectiveness of the presented approach.
\end{abstract}

Index Terms - Spinning reserve (SR); Simultaneous market clearing; Reliability preference; Value of lost load (VOLL); Cost-benefit analysis; Stochastic programming.

\section{INTRODUCTION}

Observing the security and reliability of the system is amongst the most important tasks of a power system operator. Unexpected increase of load and sudden outages of generators or transmission lines are possible events which put the security of the power system at risk. Therefore, to insure safe and reliable operation of the power system, it is essential to consider some amount of generation capacity as reserve [1]. In fact, inequality between power generation and consumption caused by failure of power system components is compensated considering adequate amount of operating reserve. Operation reserve includes spinning reserve and non-spinning reserve. Spinning reserve is considered as the difference between the capacity of active generation system and the total amount of load and losses

Published in December 28, 2017.

S. Zolfaghari is with the Electrical Engineering Department, Shahid Beheshti University, Tehran, Iran (e-mail: saeidzanjan@gmail.com).

M. H. Hashemi is with the Electrical Engineering Department, Islamic Azad University, Science and Research Branch, Tehran (Zanjan). (e-mail: m.hashemi.ir@ieee.org).

A. Zolfaghari is with the Electrical Engineering Department, University of Zanjan, Iran (e-mail: zolfaghari.azam87@gmail.com) which is able to rapidly, considering the ramp rates of generators, respond to load variation in case of failure incident.

The standard method to determine the operating reserve requirement of a system is use of deterministic criteria which considers the amount of required reserve as a certain percentage of load peak (in Spain) or equal to the capacity of largest operating generation unit (in Toronto). Also these criteria are easy to implement, they do not represent the stochastic nature of failure in power systems. To fill this gap, stochastic approaches are used in order to determine the reserve requirement of power system. Stochastic approaches estimate the amount of required reserve more realistically by combining different uncertainties in the power system such as availability of generation units and transmission system along with response rate of generators. However, lack of enough statistical data and load curves and huge amount of computations make statistical approaches more difficult to perform.

Different approaches are presented in the literature to calculate the required reserved using probabilistic criteria. The first probabilistic approach to determine capacity of reserve was PJM method which was proposed in 1963 [2]. In PJM method, forced outage rate (FOR) of generation unit is considered in determination of reserve capacity requirement. In [3]-[6] loss of load probability (LOLP) index or unit commitment risk is used. In [7] expected energy not supplied (EENS) is used instead of LOLP. Ref [8] has made use of both LOLP and EENS indices to allocate the reserve capacity and has used genetic algorithm in order to solve the optimization problem. Mixture of both deterministic and probabilistic approaches is used in [9]-[12] to provide more effective indices. In these approaches unit commitment (UC) programming is reprocessed to calculate the consumers' interruption risk level. If the calculated risk level is not acceptable, then capacity of spinning reserve is readjusted and UC is repeated until acceptable risk level is achieved.

Reference [13] introduces a UC model with security constraints which emphasizes on the simultaneous clearing of energy and ancillary services markets. In the mentioned paper, Benders decomposition method is used to decouple security-constrained unit commitment (SCUC) problem into main UC problem and sub-problem. Also, in order to solve UC problem more easy Lagrangian relaxation is used. Other methods based on reliability probabilistic indices consider the social costs of power outage as additional terms in the objective function UC problem [14],[15]. In [16] optimal reserve capacity is determined using benefit-cost analysis. Benefit of reserve allocation is estimated in terms of 
decrease in amount of EENS. On the other hand, reserve cost is the price that must be paid by the consumer to provide spinning reserve which is considered to be pay-asbid in the mentioned study. So, reserve allocation is continued until its benefit it larger than its cost. Optimization of required reserved is done in such way that minimizes the total cost of operation and expected outage cost. Also the value of lost load is not the same for all the consumers; these methods consider a single value for VOLL.

Literature review shows that consumer's decision on their desired level of reliability is not considered clearly in programming of reserve capacity allocation. In this paper, consumers declare their desired level of reliability to ISO in terms of VOLL. ISO clears the simultaneous energy and reserve market for the next hour using benefit-cost analysis and stochastic programming method.

\section{Proposed Method to Optimally Determine SPINNING RESERVE}

In this paper an hour-ahead electricity pool market has been considered in which energy and spinning reserve markets are cleared simultaneously. In this market, consumers announce their desired reliability level to ISO in terms of VOLL. The optimal spinning reserve is determined by equilibrium between expected benefit due to decrease in load shedding because of spinning reserve and the total cost of it (benefit-cost analysis) using stochastic programing. Thus, the objective function which should be minimized in in a market for the next hour is as follows:

$$
\operatorname{Min}_{P, R}\left\{\sum_{i=1}^{N_{g}}\left[C_{i} \times P_{i}+q_{i} \times R_{i}\right]+\sum_{s=0}^{N_{s}} \rho_{s} \sum_{j=1}^{N_{d}} V O L L_{j} \times U P_{j}^{s}\right\}
$$

Where: $i$ indicates the unit index (the total number of units is $\mathrm{Ng}$ ), $j$ indicates the load index (the total number of loads is $\mathrm{Nd}$ ), $s$ indicates the scenario index (the total number of scenarios is $\mathrm{Nd}), \rho s$ indicates the probability of scenario $s, C i$ indicates the bid of unit $i$ to produce energy of $\mathrm{P}$ in $(\mathrm{MW}), q_{i}$ indicates the bid of unit $i^{\text {th }}$ to provide spinning reserve of $\mathrm{R}$ in (MW), VOLL stands for value of lost load, $U P_{j}^{S}$ indicates the unsupplied power at bus $i$ in scenario $s$.

In the above objective function, the first term is related to operation cost and the second corresponds to the expected interruption cost. Solving the objective function of Equation (1) is a two-stage stochastic programming problem in which decision variables $\mathrm{P}$ and $\mathrm{R}$, are chosen in the first stage while decision variable $U P$ is determined in the second stage after the scenarios realization.

Because the interruption cost exists in the objective function and due to dependency of UP to $\mathrm{P}$ and $\mathrm{R}$ and scenarios realization, in order to make the solution faster, the problem is decoupled to a two-stage problem, a main problem (the first term) and a sub-problem (the second term). Fig.1 depicts a general view of the proposed algorithm. At the beginning, the main problem is solved using initialized reserve requirement and pre-contingency constraints to obtain preliminary generation capacity and spinning reserve capacity. These values are sent to subproblem as inputs. In the sub-problem a DC load flow problem is solved considering different scenarios and postcontingency constraints. By solving the sub-problem ISO will be capable of determination of expected interruption cost. This procedure is repeated until convergence is achieved and optimal cost is reached.

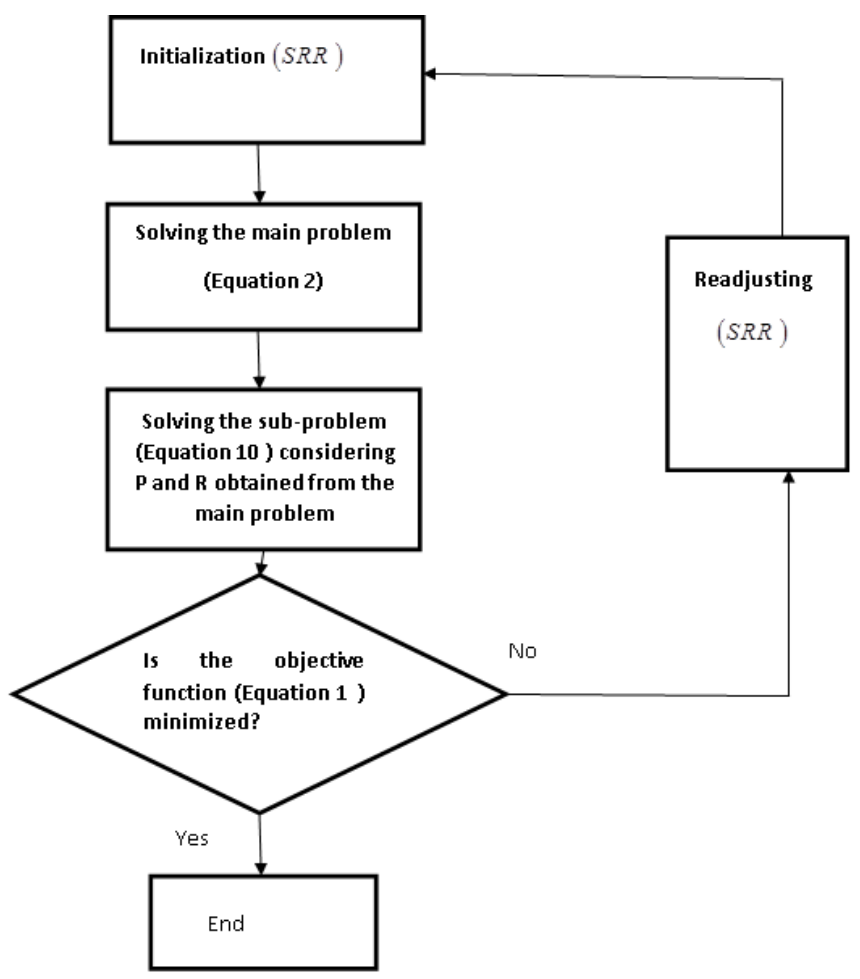

Fig. 1. Objective function optimization flowchart

\section{MAIN PROBLEM}

The main problem is formulated as follows:

$$
\operatorname{Min}\left\{\sum_{i=1}^{N_{g}}\left[C_{i} \times P_{i}+q_{i} \times R_{i}\right]\right\}
$$

In which the following constraints must be observed in order to insure the safe operation of power system:

$$
\begin{aligned}
& \sum_{i=1}^{N_{g}} P_{i}=\sum_{j=1}^{N_{d}} P_{d j} \\
& P_{i}+R_{i} \leq P_{i_{-} \max } \\
& P_{i_{-} \min } \leq P_{i}
\end{aligned}
$$

$\sum_{i=1}^{N_{g}} R_{i} \geq S R R$

$B \theta=P_{i}-P_{d}$

$P_{l}=P_{m n}=\frac{\theta_{m}-\theta_{n}}{\mathrm{X}_{m n}}$

$P_{l_{-} \text {min }} \leq P_{l} \leq P_{l_{-} \text {max }}$ 
The first constraint represents the generation-consumption balance and the other three constraints correspond the DC load flow problem.

\section{Sub-PRoblem}

As it is mentioned before, the sub-problem is a DC load flow problem which is solved considering the postcontingency constraints. Solving the sub-problem ISO will be able to determine the forced outage of generating units and transmission lines, unsupplied loads and consequently expected cost of interruptions for each scenario considering the unit commitment and spinning reserve scheduled in the main problem. The sub-problem is formulated as follows:

$$
\operatorname{Min}\left\{\sum_{s=0}^{N_{s}} \rho_{s} \sum_{j=1}^{N_{d}} V O L L_{j} \times U P_{j}^{s}\right\}
$$

Individual outage of generation units and transmission lines are the considered scenarios in the sub-problem. The probability of each scenario is obtained using FOR indices of the system components as follows:

$$
\rho_{s}=\prod_{c=1}^{n_{s}} F O R_{c} \times \prod_{c=1}^{n_{t}-n_{s}}\left(1-F O R_{c}\right)
$$

Where $n_{t}$ is the total number of system components and $n_{s}$ is the number of components that experienced forced outage in scenario $s$. The probability corresponding to normal operation of the system (i.e. no contingency) is calculated using the following equation:

$$
\rho_{0}=1-\sum_{s=1}^{N_{s}} \rho_{s}
$$

To calculate the unsupplied load, scheduled generation capacity of each unit and also spinning reserve allocation of units which are obtained from the main problem are necessary. It is assumed that the reserve capacity of each unit acts as a separate synchronized unit besides it.

In other words, reserve capacity of a unit is modeled as a generation unit besides it which provides power of $0<R<R^{\max }$. Note that $R^{\max }$ is obtained from solution of the main problem. Fig. 2 illustrates the model of spinning reserve providing units in connection to the grid.

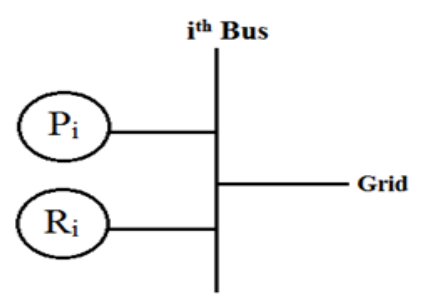

Fig 2. Model of spinning reserve providing units in connection to the grid

DC load flow formulation is used to estimate the amount of energy not supplied. Each load is modeled as an imaginary unit with maximum capacity equal to the power of the load and bid price of the corresponding declared VOLL. So, there are three types of generators in the formulation of DC load flow problem, including imaginary generators, energy providing generators and spinning reserve providing generators.

Constraints corresponding to the sub-problem are given as follows:

$$
\begin{aligned}
& \sum_{i=1}^{N_{g}} P_{i}+\sum_{j=1}^{N_{d}} U P_{j}^{s}=\sum_{j=1}^{N_{d}} P_{d j} \\
& U P_{j_{-} \text {min }}^{s} \leq U P_{j}^{s} \leq U P_{j_{-} \max }^{s} \\
& B^{s} \theta^{s}=P_{i}-P_{d}+U P_{j}^{s} \\
& P_{l}=P_{m n}=\frac{\theta_{m}-\theta_{n}}{\mathrm{X}_{m n}} \\
& P_{l_{-} \min }^{s} \leq P_{l}^{s} \leq P_{l_{-} \max }^{s}
\end{aligned}
$$

\section{CASE Study}

The proposed approach to determine the capacity of spinning reserve is evaluated on the IEEE 14-bus test system. Fig. 3 illustrates one-line diagram of this test system.

IEEE 14-bus test system includes 5 generators, 11 buses with energy consumption and 20 transmission lines. Data corresponding to the generators and the loads of this system are given in Table 1 and Table 2. In this paper, bids of generators for spinning reserve are considered to be $30 \%$ of their bids for energy.

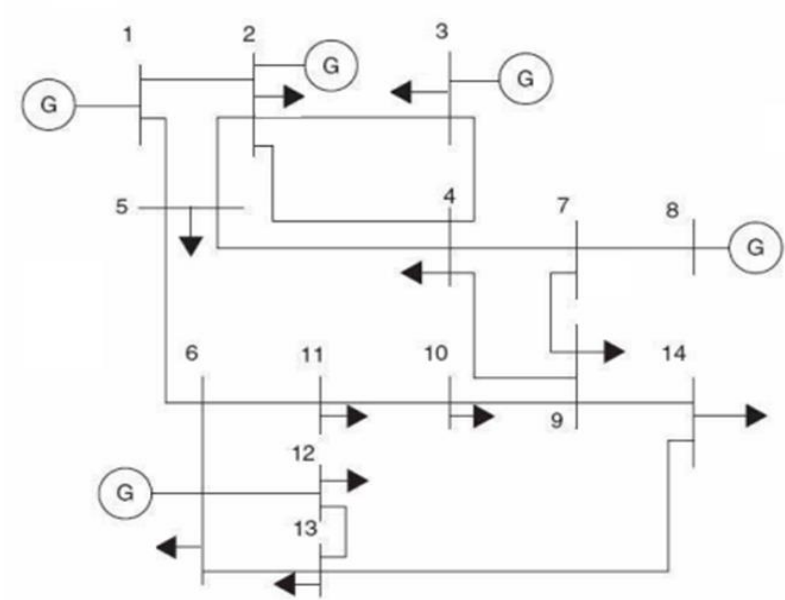

Fig 3. Single-line diagram of IEEE 14-bus test system

\begin{tabular}{ccccccc}
\multicolumn{7}{c}{ TABLE I: DATA OF IEEE 14-BUS TEST SYSTEM } \\
\hline $\begin{array}{c}\text { RAMP_UP } \\
(\mathbf{M W})\end{array}$ & $\begin{array}{c}\text { OFFER_R } \\
(\mathbf{\$} / \mathbf{M W H})\end{array}$ & $\begin{array}{c}\text { OFFER_E } \\
(\mathbf{\$} / \mathbf{M W H})\end{array}$ & $\begin{array}{c}P_{g}^{\max } \\
(\mathbf{M W})\end{array}$ & $\begin{array}{c}P_{g}^{\min } \\
(\mathbf{M W})\end{array}$ & $\mathbf{B U S}$ & UNIT \\
\hline 35 & 3 & 10 & 120 & 0 & 1 & $\mathbf{1}$ \\
40 & 3 & 10 & 150 & 0 & 2 & $\mathbf{2}$ \\
30 & 6 & 20 & 100 & 0 & 3 & $\mathbf{3}$ \\
30 & $4 / 5$ & 15 & 100 & 0 & 6 & $\mathbf{4}$ \\
25 & $13 / 5$ & 45 & 50 & 0 & 8 & $\mathbf{5}$ \\
\hline \hline
\end{tabular}

TABLE II: LOAD DATA OF IEEE 14-BUS TEST SYSTEM

\begin{tabular}{ccccc}
\hline \hline $\begin{array}{c}\text { VOLL } \\
(\$ / M W h)\end{array}$ & $\begin{array}{c}\mathrm{UP}_{\max } \\
(\mathrm{MW})\end{array}$ & $\begin{array}{c}\mathrm{UP}_{\min } \\
(\mathrm{MW})\end{array}$ & $\begin{array}{c}\mathrm{P}_{\text {Load }} \\
(\mathrm{MW})\end{array}$ & $\mathrm{BUS}$ \\
\hline- & - & - & 0 & 1 \\
600 & 38 & 0 & 38 & 2 \\
\hline \hline
\end{tabular}




\begin{tabular}{cllll}
\hline \hline 700 & 90 & 0 & 90 & 3 \\
400 & 51 & 0 & 51 & 4 \\
400 & 30 & 0 & 30 & 5 \\
600 & 16 & 0 & 16 & 6 \\
- & - & - & 0 & 7 \\
- & - & - & 0 & 8 \\
800 & 32 & 0 & 32 & 9 \\
400 & 12 & 0 & 12 & 10 \\
400 & 8 & 0 & 8 & 11 \\
400 & 20 & 0 & 20 & 12 \\
500 & 16 & 0 & 16 & 13 \\
400 & 16 & 0 & 16 & 14 \\
\hline \hline
\end{tabular}

$\mathrm{UP}_{\min }$ and $\mathrm{UP}_{\max }$ in tables I and II denotes the minimum and maximum possible load shedding. In this study four of the generators and transmission lines are considered to be 0.01 , the total amount of load is $329 \mathrm{MW}$, transmission lines have the normal capacity of $50 \mathrm{MW}$ and are to be overloaded up to $20 \%$ in case of contingency.

\section{Simulations AND RESUlts}

Contingencies considered in this study include the individual outage of generators and transmission lines. For the studied system simultaneous energy and spinning reserve market is cleared using the proposed method and the optimal spinning reserve is driven. As Fig. 4 illustrates, for the investigated case study, the objective function is minimized at spinning reserve equal to $807 \mathrm{MW}$. Fig. 5 shows the generators scheduled energy generation and spinning reserve allocation. As Fig. 2 depicts, due to the higher energy cost of unit 5 , this unit is less participated in energy and reserve providing. The whole capacity of unit 4 is utilized because it is close to the loads at buses $6,10,11,12$ and 13 .

To investigate the effects of considering reliability terms in the objective function three different scenarios are studied. The first scenario is the basic scenario in which the optimal spinning reserve requirement is calculated. In the second scenario it is assumed that there is no spinning reserve provided in the system. In the third scenario a deterministic amount of spinning reserve equal to $120 \mathrm{MW}$ is assumed.

EENS index related to load $j$ is obtained as:

$$
\operatorname{EENS}_{j}=\sum_{s \in S} \rho_{s} \times U P_{j}^{s}
$$

The EENS of the whole system is the summation over $E E N S_{j}$ for all the loads. Cost of load $j$ interruption is calculated as:

$$
E_{E N S C}=E E N S_{j} \times V O L L_{j}
$$

EENS index and interruption cost corresponding to each load is reported in Table III and Table IV for each case. As was expected, by increasing the spinning reserve allocation cost of EENS decreases while operation cost become higher. Also, the optimal case has the minimum total cost among scenarios, even though it does not have the minimum operation cost or EENS cost.

Looking at Table IV it is obvious that as the amount of allocated spinning reserve increases, EENS indices of loads decrease. However, decrease of EENS in loads 2, 3 and 4 is not significant. Interruption of load 2 in different scenarios is due to outage of unit G2. Due to congestion in Line 1-2 power delivered from unit G1 to load 2 is limited. So, in case of unit G2 outage some amount of load 2 is shed. Similarly, interruption in load 3 is due to outage of G3 and congestion in Line 2-3. Even in the third scenario in which unit G5 provides more energy, congestion in lines 3-4 and 47 causes some amount of load 3 to be shed. In the same way, congestion in lines 2-4 and 4-5 is the main cause of load 4 interruption. For loads 10 and 14 in the third scenario due to more participation of G5 no shedding occurs. However, in scenario 1 and 2 these loads experience interruption, while load 9 which has higher VOLL is not interrupted.

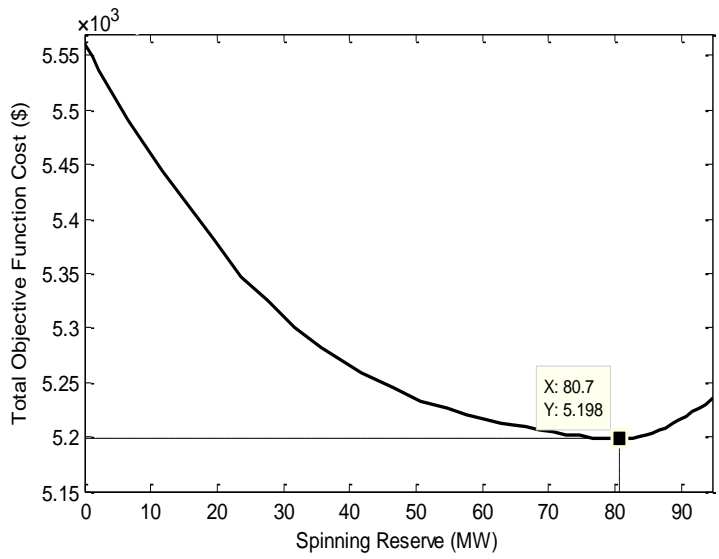

Fig. 4. Total cost as a function of spinning reserve capacity

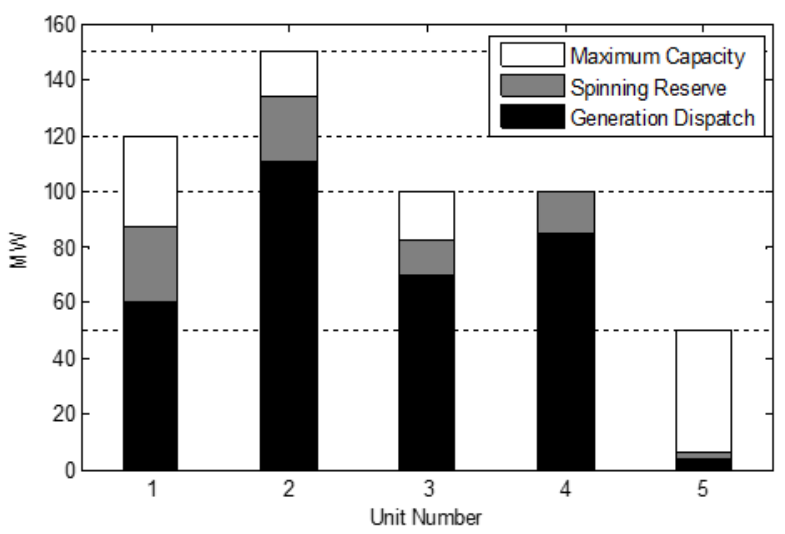

Fig. 5. Optimal energy generation and spinning reserve scheduling

TABLE III: COMPARISON OF INVESTIGATED SCENARIOS

\begin{tabular}{cccc}
\hline \hline & Scenario II & Scenario I & Scenario III \\
\hline Spinning reserve (MW) & 0 & $80 / 7$ & 120 \\
Cost of EENS (\$) & $1130 / 95$ & $326 / 874$ & $94 / 86$ \\
Operation cost (\$) & $4430 / 67$ & 4871 & $5305 / 4$ \\
Total cost $(\$)$ & $5561 / 62$ & $5197 / 874$ & $5400 / 26$ \\
\hline \hline
\end{tabular}

TABLE IV: EENS INDICES AND INTERRUPTION COSTS FOR THE INVESTIGATED SCENARIOS

\begin{tabular}{ccccccc}
\hline \hline & \multicolumn{2}{c}{ Scenario II } & \multicolumn{2}{c}{ Scenario I } & \multicolumn{2}{c}{ Scenario III } \\
Load & EENS & EENSC & EENS & EENSC & EENS & EENSC \\
& $(\mathrm{MWh})$ & $(\$)$ & $(\mathrm{MWh})$ & $(\$)$ & $(\mathrm{MWh})$ & $(\$)$ \\
\hline 2 & $0 / 2232$ & $133 / 94$ & $0 / 20064$ & $120 / 384$ & $0 / 03618$ & $21 / 71$ \\
3 & $0 / 0718$ & $50 / 29$ & $0 / 0551$ & $38 / 57$ & $0 / 02167$ & $15 / 17$ \\
4 & $0 / 6652$ & $266 / 1$ & $0 / 3071$ & $122 / 84$ & $0 / 145$ & $57 / 98$ \\
5 & $0 / 936$ & $374 / 4$ & & & - & - \\
10 & $0 / 2368$ & $94 / 74$ & $0 / 0604$ & $24 / 16$ & - & - \\
11 & $0 / 142$ & $56 / 8$ & - & - & - & - \\
12 & $0 / 2447$ & $97 / 88$ & - & $-\overline{0} 23$ & - & - \\
14 & $0 / 142$ & $56 / 8$ & $0 / 052$ & - & - \\
\hline \hline
\end{tabular}




$\begin{array}{lllllll}\text { Total } & 2 / 6617 & 1130 / 95 & 0 / 67554 & 326 / 874 & 0 / 20285 & 94 / 86\end{array}$

\section{EFFECT OF VOLL ON SPINNING RESERVE ALLOCATION}

To illustrate the effect of VOLL on amount of spinning reserve requirement and EENS, simulations are performed considering 3 different values for VOLL at buses 9 through 14. Corresponding VOLL are reported in Table 5 and simulation results are provided in Fig. 6 which illustrates the distribution of allocated spinning reserve for each generator. In the third case, because higher VOLL is chosen, G5 is scheduled to provide more energy and no interruption in loads 10 and 14 takes place. Fig. 7 shows the total reserve allocated and also EENS for each scenario. Results indicate that higher VOLL leads to more spinning reserve allocation and less EENS.

TABLE V: VOLL FOR THE INVESTIGATED SCENARIOS

\begin{tabular}{cccc}
\hline \hline Scenario 3 & Scenario 2 & Scenario 1 & \\
\hline$\% 15$ & Basic Case & $15 \%$ & VOLL \\
Increase & & Decrease & Buses 9-14 \\
\hline \hline
\end{tabular}

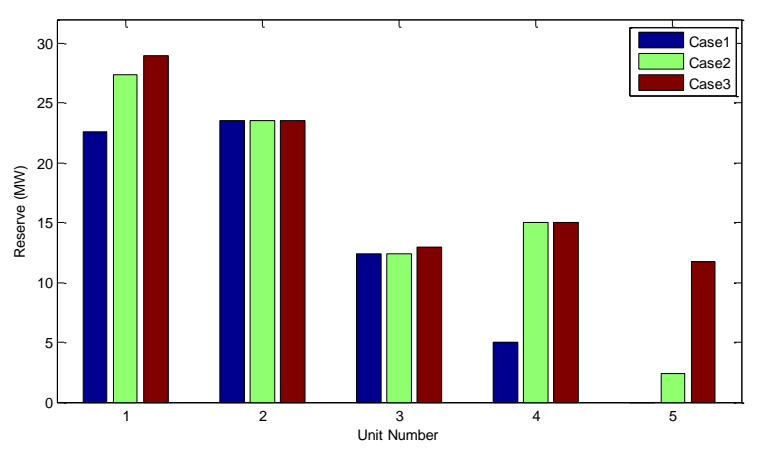

Fig. 6. Allocated spinning reserve of each unit for the investigated scenarios

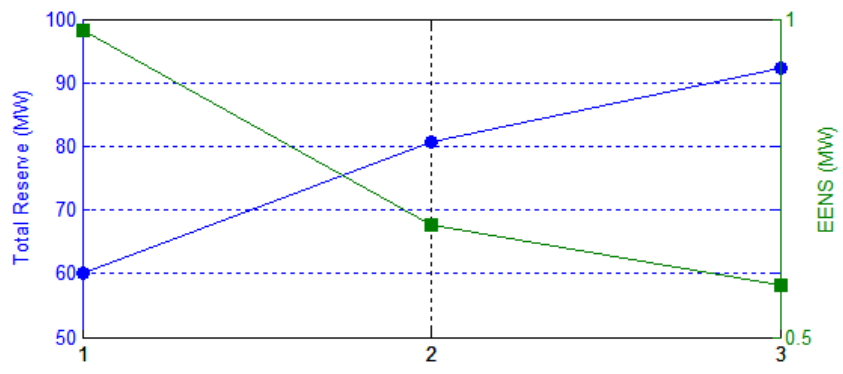

Fig 7. Total amount of reserve and EENS for the three investigated scenarios

\section{CONCLUSION}

In this paper an hour-ahead electricity pool market has been considered in which energy and spinning reserve markets are cleared simultaneously. In this market consumers declare their desirable reliability level to ISO in terms of VOLL. The optimal amount of spinning reserve is obtained by equilibrium between expected benefit of the reserve allocation due to decrease in interruption cost and the total cost of spinning reserve allocation (benefit-cost analysis) using stochastic programming.
In the proposed objective function operation cost, load interruption cost at each bus and also probability of first order contingencies are considered. Observing the rate constraints of generation units and transmission constraints in the proposed objective function the system operation security is assured as well.

The proposed approach has been evaluated on IEEE 14bus test system and the obtained results have been discussed. Results suggest that variation in the consumers' desired VOLL besides variation in total allocated spinning reserve and EENS of system causes different distribution of spinning reserve among generators.

\section{REFERENCES}

[1] Afshar, K., Ehsan, M., Fotuhi-Firuzabad, M., and Amjady, N., "Costbenefit analysis and MILP for optimal reserve capacity determination in power system," Appl Math Comput, pp. 223-229, 2008.

[2] Anstine, L. T., Burke, R. E., Holgate, R., Casey, J., John, R., and Stewart, H. G., "Application of probability methods to the determination of spinning reserve requirements for the PennsylvaniaNew Jersey-Maryland interconnection," IEEE Trans. Power App. Syst., vol. PAS-82, no. 68, pp. 720-735, Oct. 1963.

[3] Billinton, R. and Allan, R. N., Reliability Evaluation of Power System, ed., Plenun Press, USA, 1996.

[4] H. B. Gooi, D. P. Mendes, K. R. W. Bell, and D. S. Kirschen, "Optimal scheduling of spinning reserve, "IEEE Trans. Power Syst., vol. 14 , no. 4 , pp. $1485-1492$, Nov. 1999.

[5] Chattopadhyay, D., and Baldick, R., "Uint commitment with probabilistic reserve," in Proc. IEEE Power Eng. Soc. Winter Meeting, New York, vol. 1, pp. 280-285, 2002.

[6] J. Bai, H. B. Gooi, L. M. Xia, G. Strbac, and B. Venkatesh, "A probabilistic reserve market incorporating interruptible load, "IEEE Trans. Power Syst., vol. 21, no. 3, pp. 1079-1087, Aug. 2006.

[7] L. M. Xia, H. B. Gooi, and J. Bai, "A probabilistic reserve with zerosum settlement scheme,"IEEE Trans. Power Syst., vol. 20, no. 2, pp. 993-1000, May 2005.

[8] Y. Ding, P. Wang, and A. Lisnianski, “Optimal reserve management for restructured power generating systems,'Reliab. Eng. Syst. Safety, vol. 91, pp. 792-799, 2006.

[9] Billinton, R. and Fotuhi-Firuzabad, M., "A basic framework for generating system operating health analysis," IEEE Trans .Power Syst., vol. 9, no. 3, pp. 1610-1617, Aug. 1994.

[10] M. Fotuhi-Firuzabad and R. Billinton, "A security based approach for generating unit scheduling," inProc. IEEE Power Eng. Soc. Summer Meeting, Seattle, WA, Jul. 2000, pp. 195-200.

[11] M. Fotuhi-Firoozabad and M. Rashidi-nejad, "Allocation of spinning reserve among generating units using hybrid deterministic/probabilistic approach," in Proc. Large Engineering Systems Conf. Power Engineering (LESCOPE04), Halifax, NS, Canada, Jul. 2004, pp. 8187.

[12] Abiri-Jahromi, A., Fotuhi-Firuzabad, M., and Abbasi, E., "Optimal Scheduling of Spinning Reserve Based on Well-Being Model," IEEE Trans. Power Syst., vol. 22, no. 4, pp.2048-2057, November. 2007.

[13] Li, Z., and Shahidehpour, M., "Security-Constrained Unit Commitment for simultaneous clearing of Energy and Ancillary Services Markets," IEEE Trans. Power Syst., vol. 20, no. 2, pp.10791088, May. 2005.

[14] M. A. Ortega-Vazquez, D. S. Kirschen, and D. Pudjianto, "Optimising the scheduling of spinning reserve considering the cost of interrup-tions," Proc. Inst. Elect. Eng., Gen, Transm., Distrib., vol. 153, no. 5, pp. 570-575, Sep. 2006.

[15] F. Bouffard, F. D. Galiana, and A. J. Conejo, "Market-clearing with stochastic security-Part I: Formulation," IEEE Trans. Power Syst., vol. 20, no. 4, pp. 1818-1826, Nov. 2005.

[16] Wang, J., Wang, X., and Wu, Y., "Operating Reserve Model in the Power Market," IEEE Trans. Power Syst., vol. 20, no. 1, pp.223-229, Feb. 2005 